\title{
The Log-Asset Dynamic with Euler-Maruyama Scheme under Wishart Processes
}

\author{
Raphael Naryongo (iD, ${ }^{1}$ Philip Ngare, ${ }^{2}$ and Anthony Waititu ${ }^{1}$ \\ ${ }^{1}$ Department of Statistics and Actuarial Science, Jomo Kenyatta University, Juja, Kenya \\ ${ }^{2}$ School of Mathematics, University of Nairobi, Nairobi, Kenya \\ Correspondence should be addressed to Raphael Naryongo; raphael.naryongo@students.jkuat.ac.ke
}

Received 27 August 2021; Accepted 28 October 2021; Published 25 November 2021

Academic Editor: Remi Léandre

Copyright ( 2021 Raphael Naryongo et al. This is an open access article distributed under the Creative Commons Attribution License, which permits unrestricted use, distribution, and reproduction in any medium, provided the original work is properly cited.

\begin{abstract}
This article deals with Wishart process which is defined as matrix generalization of a squared Bessel process. We consider a single risky asset pricing model whose volatility is described by Wishart affine diffusion processes. The multifactor volatility specification enables this model to be flexible enough to describe the market prices for short or long maturities. The aim of the study is to derive the log-asset returns dynamic under the double Wishart stochastic volatility model. The corrected Euler-Maruyama discretization technique is applied in order to obtain the numerical solution of the log-asset return dynamic under Bi-Wishart processes. The numerical examples show the effect of the model parameters on the asset returns under the double Wishart volatility model.
\end{abstract}

\section{Introduction}

The introduction of the Heston stochastic volatility model was due to the Black and Scholes [1] model limitation of not accommodating the observable phenomenon that implied volatility of derivative products depending on strike and maturity. The Heston [2] model has been popular and widely applied in financial markets due to its flexibility, financial interpretation of parameters, and analytical tractability property since it belongs to the class of affine processes (see Filipovic and Mayerhofer [3]). The affine property allows the model to form a closed form solution of the characteristic function of the log-price, to obtain European call option price by Fourier transform inversion.

However, despite the Heston model popularity, Da Fonseca et al. [4], Christoffersen et al. [5], Ahdida and Alfonsi and Alfonsi [6], Kang et al. [7], and Gouriéroux [8] have clearly stated that the biggest weakness of the model is that it does not generate the realistic term structure of the volatility smiles. Hence, the Heston model provides too flat implied volatility surface to attain reality, yet generally implied volatility has steep curve and convexity in short maturity and tends to be linear for long maturity. This indicates that the model is not flexible enough to describe the market prices. This problem can be handled through generalizing the Heston model into a multifactor form. Using two approaches, the first is by adding jump in the stock dynamic or volatility and secondly by investigating the multifactor nature of implied volatility as in the study by Benabid et al. [9], Da Fonseca et al. [4], and Kang and Kang [10].

It is well accepted that the multifactor approach is the best one to solve the pricing problem of derivative products and volatility smile. This shows that among the multifactor models, the Wishart multidimensional stochastic volatility model (that is a matrix defined stochastic volatility model) is one of the most flexible model; this is because the term structure of the realized volatilities in this model is described by a positive semidefinite matrix-valued stochastic process. The Wishart process is defined in Bru [11] as a matrix generalization of a squared Bessel process. Da Fonseca et al. [12], Alfonsi [13], Da Fonseca et al. [4], and Benabid et al. [9] defined Wishart process as a stochastic process which is a positive semidefinite matrix-valued generalization of the square root process. The Wishart multidimensional 
stochastic volatility model found its application in finance by Gouriéroux and Sufana [14].

The aim of this study is to derive the log-asset returns dynamic under double Wishart diffusion processes, through generalization of the Heston model into multifactor nature for a single asset pricing model. That is, the asset dynamic depends on two Wishart volatility diffusion processes, with two dependence matrices describing the correlations between the asset dynamic and Wishart processes. This enables the model to be flexible enough to describe the market prices or to match the term structure of implied volatilities. This study will be of importance to investors to analyze and predict the behavior of the asset price or asset return over a period of time. The numerical solution of the log-price asset returns dynamic under the double Wishart model is obtained through the application of the corrected Euler-Maruyama discretization technique. The numerical examples demonstrate the effect of model parameters on the asset return behavior under the double Wishart volatility model.

The paper is organized as follows. In Section 2, we give definition of Wishart process, uniqueness and existence of solution, change of probability measure, and Wishart volatility model with one dependence matrix. In Section 3, we present the double Wishart stochastic volatility model, correlation structure, log-asset returns dynamic, infinitesimal generator, and Euler-Maruyama discretization scheme for the double Wishart volatility model. In Section 4, we give the numerical illustrations for log-asset returns. In Section 5, we provide conclusion and recommendation for further research work.

\section{The Wishart Process}

Definition 1. Let $W_{t}, t \geq 0$ be a $n \times n$ matrix-valued Brownian motion under the probability measure $\mathbb{Q}$. The Wishart process $\gamma_{t}$ satisfies the equation

$\mathrm{d} \gamma_{t}=\left(\beta Q Q^{T}+M \gamma_{t}+\gamma_{t} M^{T}\right) \mathrm{d} t+\sqrt{\gamma_{t}} \mathrm{~d} W_{t} Q+Q^{T} \mathrm{~d} W_{t}^{T} \sqrt{\gamma_{t}}$,

where $Q \in G L_{n}(\mathbb{R})$ is the invertible matrix, $M \in M_{n}$ is the nonpositive matrix, $\gamma_{0} \in S_{n}^{+}$is the nonnegative symmetric matrix, and $\beta$ is a real parameter. The condition $\beta>(n-1)$ is taken to ensure existence and uniqueness of the $\gamma_{t} \in S_{n}^{+}$ solution for equation (1), and eigen values of the solution are nonnegative for all $t \geq 0$ a.s $\gamma_{t} \in S_{n}^{+}$. Following Benabid et al. [9], the probability measure $\mathbb{Q}$ corresponds to a risk-neutral measure.

The infinitesimal generator of Wishart process $\gamma_{t}$ has been already studied in Bru [11], formulated as follows:

$$
\mathscr{L}_{\gamma}=\operatorname{Tr}\left[\left(\beta Q^{T} Q+M \gamma+\gamma M^{T}\right) D+2 \gamma D Q^{T} Q D\right],
$$

where the matrix differential operator $D$ is $D_{i, j}=\left(\partial / \partial \gamma_{i, j}\right)$.

2.1. The Uniqueness and Existence of the Solution of Wishart Process. The Wishart processes are affine processes defined on $S_{n}^{+}(\mathbb{R})$. Following Bru [11], La Bua and Marazzina [15], and Alfonsi [13], the results on weak and strong solution of the Wishart differential equation (1) can be shown.

Lemma 1. Let $X_{t}$ be affine process with continuous trajectories defined in $S_{n}^{+}(\mathbb{R})$, with stochastic differential equation as follows:

$$
X_{t}=X_{0}+\int_{0}^{t}\left(\bar{\alpha}+D\left(X_{s}\right)\right) \mathrm{d} s+\int_{0}^{t}\left(\sqrt{X_{s}} \mathrm{~d} W_{s} Q+Q^{T}\left(\mathrm{~d} W_{s}\right)^{T} \sqrt{X_{s}}\right)
$$

where $X_{0}, \bar{\alpha} \in S_{n}^{+}(\mathbb{R}), Q \in M_{n}(\mathbb{R})$, and $D: S_{n}^{+}(\mathbb{R}) \longrightarrow S_{n}^{+}$ $(\mathbb{R})$ is a linear transformation. The process admits a unique weak solution $\operatorname{inS}_{n}^{+}(\mathbb{R})$ if

(i) $\bar{\alpha}-(n-1) Q Q^{T} \in S_{n}^{+}(\mathbb{R})$.

(ii) For all $X_{1}, X_{2} \in S_{n}^{+}(\mathbb{R})$ such that $\operatorname{Tr}\left[\left(X_{1}\right) X_{2}\right]=$ $0 \Rightarrow \operatorname{Tr}\left[D\left(X_{1}\right) X_{2}\right] \geq 0$, where $\operatorname{Tr}[$.$] is a trace of a$ square matrix. So, $X_{0}$ contains in a set of real positive-definite matrices $S_{n}^{+}(\mathbb{R})$, with condition (i) is replaced by a stronger requirement.

(iii) $\bar{\alpha}-(n+1) Q Q^{T} \in S_{n}^{+}(\mathbb{R})$. Then, there exists a unique strong solution for equation (1) in $S_{n}^{+}(\mathbb{R})$. By observation $\bar{\alpha}=\Omega \Omega^{T}$ and $D\left[X_{0}\right]=M X_{0}+X_{0} M^{T}$ and when we assume restrictive parametrization for the deterministic part of the drift, $\Omega \Omega^{T}=\beta Q Q^{T}$. Conditions (i) and (iii) are satisfied as long as $\beta \geq n-1$ and $\beta \geq n+1$, respectively; with direct comparison, we get Wishart SDE (1) from (3). The real positive parameter $\beta$ also plays a role in Feller's condition in the univariate case.

Lemma 2. Let $\left(\mathscr{F}_{t}\right)_{t \geq 0}$ represent the filtration generated by $W_{t}, t \geq 0$. We consider continuous $\left(\mathscr{F}_{t}\right)$-adapted processes $\left(a_{t}\right)_{t \geq 0},\left(b_{t}\right)_{t \geq 0}$, and $\left(c_{t}\right)_{t \geq 0}$, respectively, valued in $M_{n}(\mathbb{R}), M_{n}(\mathbb{R})$, and $S_{n}(\mathbb{R})$ and a process $\left(M_{t}\right)_{t \geq 0}$ that admits the following semimartingale decomposition:

$$
\mathrm{d} M_{t}=c_{t} \mathrm{~d} t+b_{t} \mathrm{~d} W_{t} a_{t}+a_{t}^{T} \mathrm{~d} W_{t}^{T} b_{t}^{T} .
$$

For $i, j, m, n \in 1, \ldots, n$, the quadratic covariation of $\left(M_{t}\right)_{i, j}$ and $\left(M_{t}\right)_{m, n}$ is given as

$$
\begin{aligned}
\left\langle d\left(M_{t}\right)_{i, j}, d\left(M_{t}\right)_{m, n}\right\rangle= & {\left[\left(b_{t} b_{t}^{T}\right)_{i, m}\left(a_{t}^{T} a_{t}\right)_{j, n}+\left(b_{t} b_{t}^{T}\right)_{i, n}\left(a_{t}^{T} a_{t}\right)_{j, m}\right) } \\
& \left.+\left(b_{t} b_{t}^{T}\right)_{j, m}\left(a_{t}^{T} a_{t}\right)_{i, n}+\left(b_{t} b_{t}^{T}\right)_{j, n}\left(a_{t}^{T} a_{t}\right)_{i, m}\right] \mathrm{d} t .
\end{aligned}
$$

Proof. Clearly, we can see that the quadratic covariation (4) depends on $a_{t}$ and $b_{t}$ only through the matrices $a_{t}^{T}$ and $b_{t}^{T}$ as follows:

$$
\begin{aligned}
\mathrm{d} M_{t} & =c_{t} \mathrm{~d} t+b_{t} \mathrm{~d} W_{t} a_{t}+a_{t}^{T} \mathrm{~d} W_{t}^{T} b_{t}^{T} \\
& =c_{t} \mathrm{~d} t+\sum_{k, l=1}^{n}\left(\left(b_{t}\right)_{i, k}\left(a_{t}\right)_{l, j}+\left(b_{t}\right)_{j, k}\left(a_{t}\right)_{l, i}\right)\left(\mathrm{d} W_{t}\right)_{k, l} .
\end{aligned}
$$


The quadratic covariation is calculated as follows:

$$
\begin{aligned}
\left\langle d\left(M_{t}\right)_{i, j}, d\left(M_{t}\right)_{m, n}\right\rangle= & \sum_{k, l=1}^{n}\left(\left(b_{t}\right)_{i, k}\left(a_{t}\right)_{l, j}+\left(b_{t}\right)_{j, k}\left(a_{t}\right)_{l, i}\right)\left(\left(b_{t}\right)_{m, k}\left(a_{t}\right)_{l, n}+\left(b_{t}\right)_{n, k}\left(a_{t}\right)_{l, i m}\right) \mathrm{d} t \\
= & \sum_{k, l=1}^{n}\left(b_{t}\right)_{i, k}\left(b_{t}\right)_{m, k}\left(a_{t}\right)_{l, n}\left(a_{t}\right)_{l, j}+\sum_{k, l=1}^{n}\left(b_{t}\right)_{i, k}\left(b_{t}\right)_{n, k}\left(a_{t}\right)_{l, m}\left(a_{t}\right)_{l, j} \\
& +\sum_{k, l=1}^{n}\left(b_{t}\right)_{j, k}\left(b_{t}\right)_{m, k}\left(a_{t}\right)_{l, n}\left(a_{t}\right)_{l, i}+\sum_{k, l=1}^{n}\left(b_{t}\right)_{j, k}\left(b_{t}\right)_{n, k}\left(a_{t}\right)_{l, m}\left(a_{t}\right)_{l, i} \\
= & \left(b_{t} b_{t}^{T}\right)_{i, m}\left(a_{t}^{T} a_{t}^{T}\right)_{n, j}+\left(b_{t} b_{t}^{T}\right)_{i, n}\left(a_{t}^{T} a_{t}^{T}\right)_{m, j} \\
& +\left(b_{t} b_{t}^{T}\right)_{j, m}\left(a_{t}^{T} a_{t}^{T}\right)_{n, i}+\left(b_{t} b_{t}^{T}\right)_{j, n}\left(a_{t}^{T} a_{t}^{T}\right)_{m, i}
\end{aligned}
$$

Particularly $M_{t}$ can be written in form of trace as follows:

$$
\mathrm{d} \operatorname{Tr}\left(M_{t}\right)=\operatorname{Tr}\left(c_{t}\right) \mathrm{d} t+2 \operatorname{Tr}\left(a_{t}^{T} b_{t} \mathrm{~d} W_{t}\right) .
$$

\subsection{Link between Wishart Processes and Ornstein-Uhlenbeck} Processes. The best way to generate a Wishart process is to substitute the Gaussian vector $X_{i}$ in the definition of a Wishart distribution with Ornstein-Uhlenbeck processes $X_{i, t}$; this implies that $\beta$ is an integer. Considering $\beta$-independent $\mathrm{n}$-dimensional Ornstein-Uhlenbeck processes, $\mathrm{d} X_{k, t}=M X_{k, t} \mathrm{~d} t+Q^{T} \mathrm{~d} W_{k, t}$ and $\gamma_{t}:=\sum_{k=1}^{\beta} X_{k, t} X_{k, t}^{T}$ (see Benabid et al. [9]).

Here, $\mathrm{d} \gamma(t)=\gamma(t+\mathrm{d} t)-\gamma(t)$; then, the process $\gamma_{t}$ is a Wishart process with the following stochastic differential equation:

$$
\mathrm{d} \gamma_{t}=\left(\beta Q Q^{T}+M \gamma_{t}+\gamma_{t} M^{T}\right) \mathrm{d} t+\sqrt{\gamma_{t}} \mathrm{~d} W_{t} Q+Q^{T} \mathrm{~d} W_{t}^{T} \sqrt{\gamma_{t}},
$$

where $W$ a matrix-valued Brownian motion, obtained by

$$
\sqrt{\gamma_{t}} \mathrm{~d} W_{t}=\sum_{k=1}^{\beta} X_{k, t} \mathrm{~d} W_{k, t}^{T},
$$

where the matrix $M$ is taken as the mean reversion parameter of the Wishart process and $Q$ is the volatility parameter.

2.3. Change of the Probability Measure. From the mathematics point of view, it is necessary to describe the change of the probability measure to allow a change of the drift in the dynamics process. In financial application, especially in the practical aspects, Wishart process has to be simulated in its general form with $\beta \geq n+1$, such that $\beta \in \mathbb{R}$. The function permits to write $K=\beta+2 \lambda$ with $\beta=K \geq n+1$ and $\lambda$ real number with $0 \leq \lambda \leq 1 / 2$.

The goal is to find a change of probability measure in order to change the generalized Wishart diffusion process into the simple one, where $\beta$ is an integer. Therefore, the new probability measure $\mathbb{P}$, following Benabid and Bjork can be expressed as follows.

Theorem 1. Let $q=\beta+\lambda-n-1$. If $h_{T}(\mathbb{Q}, \mathbb{P})=d \mathbb{Q} / d \mathbb{P}$ defines the Radon-Nikodym derivative of $\mathbb{Q}$ with respect to $\mathbb{P}$, then

$$
h_{T}(\mathbb{Q}, \mathbb{P})=\left(\frac{\operatorname{det}\left(\gamma_{T}\right)}{\operatorname{det}\left(\gamma_{0}\right)}\right)^{\lambda / 2} \exp (-\lambda T \operatorname{Tr}(M)) \exp \left[\frac{-\lambda}{2} q \int_{0}^{T} \operatorname{Tr}\left(\gamma_{s}^{-1} Q^{T} Q\right) \mathrm{d} s\right] .
$$

Proof. The probability measure $\mathbb{P}$ can be specified through an exponential martingale.

$$
\frac{\mathrm{d} \mathbb{Q}}{\mathrm{d} \mathbb{P}}=\exp \left\{\lambda \int_{0}^{T} \operatorname{Tr}\left[\sqrt{\gamma_{s}^{-1}} \mathrm{~d} W_{s} Q\right]-\frac{\lambda^{2}}{2} \int_{0}^{T} \operatorname{Tr}\left(\gamma_{s}^{-1} Q^{T} Q\right) \mathrm{d} s\right\} .
$$

We define the new process as follows:

$$
W_{t}=\widetilde{W}_{t}+\lambda \int_{0}^{t} \sqrt{\gamma_{s}^{-1}} Q^{T} \mathrm{~d} s
$$

We check using Girsanov theorem that $W$ is a matrixvalued Brownian motion under the probability measure $\mathbb{P}$ while the dynamics of Wishart diffusion process under the probability measure $\mathbb{P}$ is as follows:

$$
\mathrm{d} \gamma_{t}=\left(\beta Q Q^{T}+M \gamma_{t}+\gamma_{t} M^{T}\right) \mathrm{d} t+\sqrt{\gamma_{t}} \mathrm{~d} W_{t} Q+Q^{T} \mathrm{~d} W_{t}^{T} \sqrt{\gamma_{t}},
$$


where

$$
\begin{aligned}
W_{t} & =\widetilde{W}_{t}+\lambda \int_{0}^{t} \sqrt{\gamma_{s}^{-1}} Q^{T} \mathrm{~d} s, \\
\mathrm{~d} W_{t} & =\mathrm{d} \widetilde{W}_{t}+\lambda \sqrt{\gamma_{t}^{-1}} Q^{T} \mathrm{~d} t .
\end{aligned}
$$

$$
\mathrm{d} \gamma_{t}=\left(\beta Q Q^{T}+M \gamma_{t}+\gamma_{t} M^{T}\right) \mathrm{d} t+\sqrt{\gamma_{t}}\left(\mathrm{~d} \widetilde{W_{t}}+\lambda \sqrt{\gamma_{t}^{-1}} Q^{T} \mathrm{~d} t\right) Q+Q^{T}\left(\mathrm{~d} \widetilde{W_{t}}+\lambda \sqrt{\gamma_{t}^{-1}} Q^{T} \mathrm{~d} t\right)^{T} \sqrt{\gamma_{t}}
$$

Then,

$$
\mathrm{d} \gamma_{t}=\left((\beta+2 \lambda) Q Q^{T}+M \gamma_{t}+\gamma_{t} M^{T}\right) \mathrm{d} t+\sqrt{\gamma_{t}} \mathrm{~d} \widetilde{W_{t}} Q+Q^{T} \mathrm{~d} \widetilde{W}_{t}^{T} \sqrt{\gamma_{t}} .
$$

The Radon-Nikodym derivative is computed using the determinant dynamics.

$$
\begin{array}{r}
\int_{0}^{T} d \log \left(\operatorname{det} \gamma_{s}\right) \int_{0}^{T}\left[(\beta-n-1) \operatorname{Tr}\left(\gamma_{s}^{-1} Q^{T} Q\right)+2 \operatorname{Tr}(M)\right] \mathrm{d} s+2 \int_{0}^{T} \operatorname{Tr}\left[\sqrt{\gamma_{s}^{-1}} \mathrm{~d} W_{s} Q\right] \\
\log \left(\frac{\operatorname{det} \gamma_{T}}{\operatorname{det} \gamma_{0}}\right)=2 \operatorname{Tr}(M)+\int_{0}^{T}(\beta-n-1) \operatorname{Tr}\left(\gamma_{s}^{-1} Q^{T} Q\right) \mathrm{d} s+2 \int_{0}^{T} \operatorname{Tr}\left[\sqrt{\gamma_{s}^{-1}} \mathrm{~d} W_{s} Q\right] \\
\log \left(\frac{\operatorname{det} \gamma_{T}}{\operatorname{det} \gamma_{0}}\right)^{\lambda / 2}=\lambda T \operatorname{Tr}(M)+\frac{\lambda}{2} \int_{0}^{T}(\beta-n-1) \operatorname{Tr}\left(\gamma_{s}^{-1} Q^{T} Q\right) \mathrm{d} s+\lambda \int_{0}^{T} \operatorname{Tr}\left[\sqrt{\gamma_{s}^{-1}} \mathrm{~d} W_{s} Q\right], \\
\exp \left\{\lambda \int_{0}^{T} \operatorname{Tr}\left[\sqrt{\gamma_{s}^{-1}} \mathrm{~d} W_{s} Q\right]\right\}=\left(\frac{\operatorname{det} \gamma_{T}}{\operatorname{det} \gamma_{0}}\right)^{\lambda / 2} \exp \{-\lambda T \operatorname{Tr}(M)\} \exp \left\{-\frac{\lambda}{2} \int_{0}^{T}(\beta-n-1) \operatorname{Tr}\left(\gamma_{s}^{-1} Q^{T} Q\right) \mathrm{d} s\right\} .
\end{array}
$$

From equation (12), the new measure with $\widetilde{W}$ is given by

$$
\begin{aligned}
\frac{\mathrm{d} \mathbb{Q}}{\mathrm{d} \mathbb{P}}= & \exp \left\{\lambda \int_{0}^{T} \operatorname{Tr}\left[\gamma_{s}^{-1} \mathrm{~d} \widetilde{W}_{s} Q\right]\right\} \exp \left\{-\frac{\lambda^{2}}{2} \int_{0}^{T} \operatorname{Tr}\left(\gamma_{s}^{-1} Q^{T} Q\right) \mathrm{d} s\right\} \\
& . \exp \left\{-\frac{\lambda}{2} \int_{0}^{T}(\beta+2 \lambda-n-1) \operatorname{Tr}\left(\gamma_{s}^{-1} Q^{T} Q\right) \mathrm{d} s\right\} \exp \left\{-\frac{\lambda^{2}}{2} \int_{0}^{T} \operatorname{Tr}\left(\gamma_{s}^{-1} Q^{T} Q\right) \mathrm{d} s\right\} \\
= & \left(\frac{\operatorname{det} \gamma_{T}}{\operatorname{det} \gamma_{0}}\right)^{\lambda / 2} \exp \{-\lambda T \operatorname{Tr}(M)\} \exp \left\{\left[-\frac{\lambda}{2}(\beta+2 \lambda-n-1)-\frac{\lambda^{2}}{2}\right] \int_{0}^{T} \operatorname{Tr}\left(\gamma_{s}^{-1} Q^{T} Q\right) \mathrm{d} s\right\} \\
= & \left(\frac{\operatorname{det} \gamma_{T}}{\operatorname{det} \gamma_{0}}\right)^{\lambda / 2} \exp \{-\lambda T \operatorname{Tr}(M)\} \exp \left\{-\frac{\lambda}{2}(\beta+\lambda-n-1) \int_{0}^{T} \operatorname{Tr}\left(\gamma_{s}^{-1} Q^{T} Q\right) \mathrm{d} s\right\} .
\end{aligned}
$$

The change of the probability measure is obtained. 
2.4. Wishart Volatility Model. In the study by Da Fonseca et al. [4] and Benabid et al. [9], under the risk-neutral probability measure and arbitrage-free financial market, the risky asset price dynamic and its volatility process are as follows:

$$
\begin{aligned}
\frac{\mathrm{d} S(t)}{S(t)} & =\mu \mathrm{d} t+\operatorname{Tr}\left[\sqrt{\gamma_{t}} \mathrm{~d} Z_{t}\right], S_{0}=s, \\
\mathrm{~d} \gamma_{t} & =\left(\beta Q Q^{T}+M \gamma_{t}+\gamma_{t} M^{T}\right) \mathrm{d} t+\sqrt{\gamma_{t}} \mathrm{~d} W_{t} Q+Q^{T} \mathrm{~d} W_{t}^{T} \sqrt{\gamma_{t}}, \quad \gamma_{0}=\gamma,
\end{aligned}
$$

where $\mu$ represents the risk free interest rate, $\operatorname{Tr}$ is the trace operator, $Z \in M_{n}$ is a matrix Brownian motion under the risk-neutral measure, and $\gamma_{t}$ belongs to the set of symmetric $n \times n$ positive-definite matrices as well as its square root $\sqrt{\gamma_{t}}$. We observe that volatility of the risky asset is the trace of the matrix $\gamma_{t}$, with $\Omega, M, Q \in M_{n}$, and $W_{t} \in M_{n}$ is a matrix Brownian motion.

The dynamic of the Wishart process (see Bru [11]) denotes a matrix analogue of the square root mean-reverting process. To ensure strict positivity and the typical meanreverting feature of the volatility, matrix $M$ is considered to be negative semidefinite, with the real parameter $\beta>n-1$, for condition of uniqueness and existence of the solution of the dynamics of the Wishart processes.

\section{Presentation of Double Wishart Model in Stock Market}

This section introduces a proposed novel model, the multifactor model with two Wishart processes or double Wishart stochastic volatility model with two dependence matrices. The model takes two underlying volatility components defined as the trace of a Wishart process. However, following Naryongo et al. [17], the diagonal components of the Wishart matrices will be the factors guiding the dynamics of volatilities.

Under arbitrage-free financial market and under the risk-neutral probability measure, we consider the following risky asset dynamic:

$$
\frac{\mathrm{d} S_{t}}{S_{t}}=\mu \mathrm{d} t+\operatorname{Tr}\left[\sqrt{\gamma}_{1, t} \mathrm{~d} Z_{t}^{\gamma_{1}}+\sqrt{\gamma}_{2, t} \mathrm{~d} Z_{t}^{\gamma_{2}}\right],
$$

where the quadratic variations are as follows:

$$
\begin{aligned}
& \mathrm{d} \gamma_{1, t}=\left(\beta_{1} Q_{1} Q_{1}^{T}+M_{1} \gamma_{1, t}+\gamma_{1, t} M_{1}^{T}\right) \mathrm{d} t+\sqrt{\gamma}_{1, t} \mathrm{~d} W_{t}^{\gamma_{1}} Q_{1}+Q_{1}^{T}\left(\mathrm{~d} W_{t}^{\gamma_{1}}\right)^{T} \sqrt{\gamma_{1, t}}, \quad \gamma_{1,0}=\gamma_{1}, \\
& \mathrm{~d} \gamma_{2, t}=\left(\beta_{2} Q_{2} Q_{2}^{T}+M_{2} \gamma_{2, t}+\gamma_{2, t} M_{2}^{T}\right) \mathrm{d} t+\sqrt{\gamma}_{2, t} \mathrm{~d} W_{t}^{\gamma_{2}} Q_{2}+Q_{2}^{T}\left(\mathrm{~d} W_{t}^{\gamma_{2}}\right)^{T} \sqrt{\gamma_{2, t}}, \quad \gamma_{2,0}=\gamma_{2} \text {, }
\end{aligned}
$$

where $\beta_{1}$ and $\beta_{2}$ are real parameters such that $\beta_{1}, \beta_{2}>n-1$, $Q_{1}, Q_{2} M_{1}, M_{2} \in M_{n}, \quad Q$ is invertible matrix, and $W_{t}^{\gamma_{1}}, W_{t}^{\gamma_{2}} \in M_{n}$ are matrices Brownian motions, and also $Z_{t}^{\gamma_{1}}, Z_{t}^{\gamma_{2}} \in M_{n}$.

Lemma 3. The correlation between the Brownian matrices of the stock price dynamic and the Brownian matrices of the Wishart processes is given as

$$
\begin{gathered}
\rho_{1, t}=\frac{\operatorname{Tr}\left(R_{1}^{T} Q_{1} \gamma_{1, t}\right)}{\sqrt{\operatorname{Tr}\left(\gamma_{1, t}\right)} \sqrt{\operatorname{Tr}\left(Q_{1}^{T} Q_{1} \gamma_{1, t}\right)}}, \\
\rho_{2, t}=\frac{\operatorname{Tr}\left(R_{2}^{T} Q_{2} \gamma_{2, t}\right)}{\sqrt{\operatorname{Tr}\left(\gamma_{2, t}\right)} \sqrt{\operatorname{Tr}\left(Q_{2}^{T} Q_{2} \gamma_{2, t}\right)}} .
\end{gathered}
$$

Proof.

$$
\begin{aligned}
\frac{\mathrm{d} S_{t}}{S_{t}} & =r \mathrm{~d} t+\operatorname{Tr}\left[\sqrt{\gamma_{1, t}} \mathrm{~d} Z_{t}^{\gamma_{1}}+\sqrt{\gamma_{2, t}} \mathrm{~d} Z_{t}^{\gamma_{2}}\right] \\
& =r \mathrm{~d} t+\sqrt{\operatorname{Tr}\left(\gamma_{1, t}\right)} \frac{\operatorname{Tr}\left(\sqrt{\gamma_{1, t}} \mathrm{~d} Z_{t}^{\gamma_{1}}\right)}{\sqrt{\operatorname{Tr}\left(\gamma_{1, t}\right)}}+\sqrt{\operatorname{Tr}\left(\gamma_{2, t}\right)} \frac{\operatorname{Tr}\left(\sqrt{\gamma_{2, t}} \mathrm{~d} Z_{t}^{\gamma_{2}}\right)}{\sqrt{\operatorname{Tr}\left(\gamma_{2, t}\right)}} \\
& =r \mathrm{~d} t+\sqrt{\operatorname{Tr}\left(\gamma_{1, t}\right)} \mathrm{d} X_{t}^{\gamma_{1}}+\sqrt{\gamma_{2, t}} \mathrm{~d} X_{t}^{\gamma_{2}}
\end{aligned}
$$

where $X_{t}^{\gamma_{1}}$ and $X_{t}^{\gamma_{2}}$ are standard Brownian motions (see proof in Appendix), and also considering the trace of the dynamics of Wishart volatility process (21), we get

$$
\begin{aligned}
& \mathrm{d} \operatorname{Tr}\left(\gamma_{1, t}\right)=\left(\left(\beta_{1} \operatorname{Tr}\left(Q_{1}^{T} Q_{1}\right)\right)+2 \operatorname{Tr}\left(M_{1} \gamma_{1, t}\right)\right) \mathrm{d} t+2 \operatorname{Tr}\left(Q_{1} \mathrm{~d} W_{t}^{\gamma_{1}} \sqrt{\gamma_{1, t}}\right), \\
& \mathrm{d} \operatorname{Tr}\left(\gamma_{2, t}\right)=\left(\left(\beta_{2} \operatorname{Tr}\left(Q_{2}^{T} Q_{2}\right)\right)+2 \operatorname{Tr}\left(M_{2} \gamma_{2, t}\right)\right) \mathrm{d} t+2 \operatorname{Tr}\left(Q_{2} \mathrm{~d} W_{t}^{\gamma_{2}} \sqrt{\gamma_{2, t}}\right) .
\end{aligned}
$$

These processes can still be presented in the form as follows: 


$$
\begin{aligned}
& \mathrm{d} \operatorname{Tr}\left(\gamma_{1, t}\right)=\left(\left(\beta_{1} \operatorname{Tr}\left(Q_{1}^{T} Q_{1}\right)\right)+2 \operatorname{Tr}\left(M_{1} \gamma_{1, t}\right)\right) \mathrm{d} t+2 \sqrt{\operatorname{Tr}\left(Q_{1}^{T} Q_{1} \gamma_{1, t}\right)} \frac{\operatorname{Tr}\left(Q_{1} \mathrm{~d} W_{t}^{\gamma_{1}} \sqrt{\gamma_{1, t}}\right)}{\sqrt{\operatorname{Tr}\left(Q_{1}^{T} Q_{1} \gamma_{1, t}\right)}}, \\
& \mathrm{d} \operatorname{Tr}\left(\gamma_{2, t}\right)=\left(\left(\beta_{2} \operatorname{Tr}\left(Q_{2}^{T} Q_{2}\right)\right)+2 \operatorname{Tr}\left(M_{2} \gamma_{2, t}\right)\right) \mathrm{d} t+2 \sqrt{\operatorname{Tr}\left(Q_{2}^{T} Q_{1} \gamma_{2, t}\right)} \frac{\operatorname{Tr}\left(Q_{2} \mathrm{~d} W_{t}^{\gamma_{2}} \sqrt{\gamma_{2, t}}\right)}{\sqrt{\operatorname{Tr}\left(Q_{2}^{T} Q_{2} \gamma_{2, t}\right)}},
\end{aligned}
$$

where $\xi_{t}$ and $\eta_{t}$ are Brownian motions (see proof in Appendix), such that

$$
\begin{aligned}
& \mathrm{d} \operatorname{Tr}\left(\gamma_{1, t}\right)=\left(\left(\beta_{1} \operatorname{Tr}\left(Q_{1}^{T} Q_{1}\right)\right)+2 \operatorname{Tr}\left(M_{1} \gamma_{1, t}\right)\right) \mathrm{d} t+2 \sqrt{\operatorname{Tr}\left(Q_{1}^{T} Q_{1} \gamma_{1, t}\right) \mathrm{d} \xi_{t}}, \\
& \mathrm{~d} \operatorname{Tr}\left(\gamma_{2, t}\right)=\left(\left(\beta_{2} \operatorname{Tr}\left(Q_{2}^{T} Q_{2}\right)\right)+2 \operatorname{Tr}\left(M_{2} \gamma_{2, t}\right)\right) \mathrm{d} t+2 \sqrt{\operatorname{Tr}\left(Q_{2}^{T} Q_{2} \gamma_{2, t}\right) \mathrm{d} \eta_{t}} .
\end{aligned}
$$

We now determine the covariation of the stock price and Wishart processes:

$$
\begin{aligned}
\operatorname{Cov}_{t}\left(\mathrm{~d} X_{t}^{\gamma_{1}}, \mathrm{~d} \xi_{t}\right) & =\operatorname{Cov}_{t}\left(\frac{\operatorname{Tr}\left(\sqrt{\gamma_{1, t}} \mathrm{~d} Z_{t}^{\gamma_{1}}\right)}{\sqrt{\operatorname{Tr}\left(\gamma_{1, t}\right)}}, \frac{\operatorname{Tr}\left(Q_{1} \mathrm{~d} W_{t}^{\gamma_{1}} \sqrt{\gamma_{1, t}}\right)}{\sqrt{\operatorname{Tr}\left(Q_{1}^{T} Q_{1} \gamma_{1, t}\right)}}\right) \\
& =\mathbb{E}_{t}\left(\frac{\operatorname{Tr}\left(\sqrt{\gamma_{1, t}} \mathrm{~d} W_{t}^{\gamma_{1}} R_{1}^{T}\right)}{\sqrt{\operatorname{Tr}\left(\gamma_{1, t}\right)}} \frac{\operatorname{Tr}\left(Q_{1} \mathrm{~d} W_{t}^{\gamma_{1}} \sqrt{\gamma_{1, t}}\right)}{\sqrt{\operatorname{Tr}\left(Q_{1}^{T} Q_{1} \gamma_{1, t}\right)}}\right)=\frac{\sum_{i j} \operatorname{Cov}_{t}\left(e_{i}^{T} \sqrt{\gamma_{1, t}} \mathrm{~d} W_{t}^{\gamma_{1}} R_{1}^{T} e_{i}, e_{j}^{T} Q_{1} \mathrm{~d} W_{t}^{\gamma_{1}} \sqrt{\gamma_{1, t}} e_{j}\right)}{\sqrt{\operatorname{Tr}\left(\gamma_{1, t}\right)} \sqrt{\operatorname{Tr}\left(Q_{1}^{T} Q_{1} \gamma_{1, t}\right)}} \\
& =\frac{\sum_{i j} \mathbb{E}_{t}\left(e_{i}^{T} \sqrt{\gamma_{1, t}} \mathrm{~d} W_{t}^{\gamma_{1}} R_{1}^{T} e_{i} e_{j}^{T} Q_{1}^{T}\left(\mathrm{~d} W_{t}^{\gamma_{1}}\right)^{T} \sqrt{\gamma_{1, t}} e_{j}\right)}{\sqrt{\operatorname{Tr}\left(\gamma_{1, t}\right)} \sqrt{\operatorname{Tr}\left(Q_{1}^{T} Q_{1} \gamma_{1, t}\right)}} \\
& =\frac{\sum_{i j} e_{i}^{T} \sqrt{\gamma_{1, t}} \operatorname{Tr}\left(R_{1}^{T} e_{i} e_{j}^{T} Q_{1}^{T}\right) \sqrt{\gamma_{1, t}} e_{j} \mathrm{~d} t}{\sqrt{\operatorname{Tr}\left(\gamma_{1, t}\right)} \sqrt{\operatorname{Tr}\left(Q_{1}^{T} Q_{1} \gamma_{1, t}\right)}} \\
& =\frac{\sum_{i j} \operatorname{Tr}\left(Q_{1} R_{1} e_{i} e_{j}^{T}\right) e_{i}^{T} \gamma_{1, t} e_{j} \mathrm{~d} t}{\sqrt{\operatorname{Tr}\left(\gamma_{1, t}\right)} \sqrt{\operatorname{Tr}\left(Q_{1}^{T} Q_{1} \gamma_{1, t}\right)}} \\
& =\frac{\sum_{i j} e_{j}^{T} Q_{1} \operatorname{Re} e_{i} e_{i}^{T} \gamma_{1, t} e_{j} \mathrm{~d} t}{\sqrt{\operatorname{Tr}\left(\gamma_{1, t}\right)} \sqrt{\operatorname{Tr}\left(Q_{1}^{T} Q_{1} \gamma_{1, t}\right)}} \\
& =\frac{\sum_{i j} e_{j}^{T} Q_{1} R_{1} \gamma_{1, t} e_{j} \mathrm{~d} t}{\sqrt{\operatorname{Tr}\left(\gamma_{1, t}\right)} \sqrt{\operatorname{Tr}\left(Q_{1}^{T} Q_{1} \gamma_{1, t}\right)}} \\
& \frac{\operatorname{Tr}\left(R_{1}^{T} Q_{1} \gamma_{1, t}\right)}{\sqrt{\operatorname{Tr}\left(\gamma_{1, t}\right)} \sqrt{\operatorname{Tr}\left(Q_{1}^{T} Q_{1} \gamma_{1, t}\right)}}=\rho_{1, t} \cdot
\end{aligned}
$$

The same procedures can be carried out on the second differential equation to obtain the covariation as follows:

$$
\operatorname{Cov}_{t}\left(\mathrm{~d} X_{t}^{\gamma_{2}}, \mathrm{~d} \eta_{t}\right)=\frac{\operatorname{Tr}\left(R_{2}^{T} Q_{2} \gamma_{2, t}\right)}{\sqrt{\operatorname{Tr}\left(\gamma_{2, t}\right)} \sqrt{\operatorname{Tr}\left(Q_{2}^{T} Q_{2} \gamma_{2, t}\right)}}=\rho_{2, t} .
$$


The two correlations between the Brownian motions of the asset and the Brownian motions of the Wishart processes are stochastic.

3.1. The Correlation Structure of the Model. The Brownian matrices $W_{t}^{\gamma_{1}}, W_{t}^{\gamma_{2}}, Z_{t}^{\gamma_{1}}$, and $Z_{t}^{\gamma_{2}}$ can be correlated in such a way that all the (scalar) Brownian motions belonging to $i$ of $Z_{t}^{\gamma_{1}}$ and $Z_{t}^{\gamma_{2}}$ and corresponding Brownian motions of the column $j$ of $W_{t}^{\gamma_{1}}$ and $W_{t}^{\gamma_{2}}$ have the same correlation, say $R_{i j}^{\gamma_{1}}$ and $R_{i j}^{\gamma_{2}}$. This provides constant matrices $R_{1}, R_{2} \in M_{n}$ which describe the two correlation structures, in such a way that $Z_{t}^{\gamma_{1}}$ and $Z_{t}^{\gamma_{2}}$ presented as follows:

$$
Z_{t}^{\gamma_{k}}:=W_{t}^{\gamma_{k}} R_{k}^{T}+B_{t}^{\gamma_{k}} \sqrt{\square-R_{k} R_{k}^{T}}, \text { for } \quad k=1,2,
$$

where ? denotes the identity matrix, $T$ is the transposition, and $B_{t}^{\gamma_{1}}$ and $B_{t}^{\gamma_{2}}$ are (matrices) Brownian motion independent of $W_{t}^{\gamma_{1}}$ and $W_{t}^{\gamma_{2}}$, respectively.

Lemma 4. The correlation structure $Z_{t}^{\gamma_{1}}:=W_{t}^{\gamma_{1}} R_{1}^{T}+$ $B_{t}^{\gamma_{1}} \sqrt{\square-R_{1} R_{1}^{T}}$ is a matrix Brownian motion.

Proof. We note that $Z_{t}^{\gamma_{1}}$ is a matrix Brownian motion iff for $\alpha_{1}, \beta_{1} \in \mathbb{R}^{n}$,

$$
\operatorname{Cov}_{t}\left(\mathrm{~d} Z_{t}^{\gamma_{1}}, \mathrm{~d} Z_{t}^{\gamma_{1}} \beta_{1}\right)=\mathbb{E}_{t}\left[\left(\mathrm{~d} Z_{t}^{\gamma_{1}} \alpha_{1}\right)\left(\mathrm{d} Z_{t}^{\gamma_{1}} \beta_{1}\right)^{T}\right]=\alpha_{1}^{T} \beta_{1} I \mathrm{~d} t .
$$

Then,

$$
\begin{aligned}
\operatorname{Cov}_{t}\left(\mathrm{~d} Z_{t}^{\gamma_{1}} \alpha_{1}, \mathrm{~d} Z_{t}^{\gamma_{1}} \beta_{1}\right)= & \mathbb{E}_{t}\left[\left(\mathrm{~d} W_{t}^{\gamma_{1}} R_{1}^{T} \alpha_{1}+\mathrm{d} B_{t}^{\gamma_{1}} \sqrt{\square-R_{1} R_{1}^{T} \alpha_{1}}\right)\left(\mathrm{d} W_{t}^{\gamma_{1}} R_{1}^{T} \beta_{1}+\mathrm{d} B_{t}^{\gamma_{1}} \sqrt{\square-R_{1} R_{1}^{T} \beta_{1}}\right)\right] \\
= & \operatorname{Cov}_{t}\left(\mathrm{~d} W_{t}^{\gamma_{1}} R_{1}^{T} \alpha_{1}, \mathrm{~d} W_{t}^{\gamma_{1}} R_{1}^{T} \beta_{1}\right) \\
& +\operatorname{Cov}_{t}\left(\mathrm{~d} B_{t}^{\gamma_{1}} \sqrt{\square-R_{1} R_{1}^{T} \alpha_{1}}, \mathrm{~d} B_{t}^{\gamma_{1}} \sqrt{\square-R_{1} R_{1}^{T} \beta_{1}}\right) \\
= & \alpha_{1}^{T} R_{1} R_{1}^{T} \beta_{1} \llbracket \mathrm{d} t+\alpha_{1}^{T}\left(\llbracket-R_{1} R_{1}^{T}\right) \beta_{1} \llbracket \mathrm{d} t \\
= & \alpha_{1}^{T} \beta_{1} \rrbracket \mathrm{d} t
\end{aligned}
$$

similarly for the second dynamic process as

$$
\operatorname{Cov}_{t}\left(\mathrm{~d} Z_{t}^{\gamma_{2}} \alpha_{2}, \mathrm{~d} Z_{t}^{\gamma_{2}} \beta_{2}\right)=\alpha_{2}^{T} \beta_{2} \rrbracket \mathrm{d} t .
$$

3.2. The Log-Asset Return Dynamic under Double Wishart Model. Let $Y_{t}$ denote the log-price and the matrices $R_{1}$ and $R_{2}$ describe the correlations between the Brownian of the asset price process and those of the Wishart processes.

Proposition 1. The log-price process $Y_{t}=\log \left(S_{t}\right)$ under the double Wishart volatility model is as follows:

$$
\mathrm{d} Y_{t}=\left(r-\frac{1}{2} \operatorname{Tr}\left[\gamma_{1, t}+\gamma_{2, t}\right]\right) \mathrm{d} t+\operatorname{Tr}\left[\sqrt{\gamma_{1, t}} \mathrm{~d} Z_{t}^{\gamma_{1}}+\sqrt{\gamma_{2, t}} \mathrm{~d} Z_{t}^{\gamma_{2}}\right] .
$$

Proof. Let $Y_{t}=\log \left(S_{t}\right)$, the asset dynamic is given as follows:

$$
\frac{\mathrm{d} S(t)}{S(t)}=r \mathrm{~d} t+\operatorname{Tr}\left[\sqrt{\gamma_{1, t}} \mathrm{~d} Z_{t}^{\gamma_{1}}+\sqrt{\gamma_{2, t}} \mathrm{~d} Z_{t}^{\gamma_{2}}\right] .
$$

By applying Ito's formula on $Y_{t}$ (see Björk [16] and Shreve [18]), we get

$$
\mathrm{d} Y_{t}=d \log \left(S_{t}\right)=\frac{\mathrm{d} S_{t}}{S_{t}}-\frac{1}{2} \frac{\left(\mathrm{d} S_{t}\right)^{2}}{S_{t}^{2}} .
$$

Replacing the asset process (35) in the derivative equation (36) of $Y_{t}$,

$$
\begin{aligned}
\mathrm{d} Y(t) & =r \mathrm{~d} t+\operatorname{Tr}\left[\sqrt{\gamma_{1, t}} \mathrm{~d} Z_{t}^{\gamma_{1}}+\sqrt{\gamma_{2, t}} \mathrm{~d} Z_{t}^{\gamma_{2}}\right]-\frac{1}{2} \operatorname{Tr}\left[\gamma_{1, t}+\gamma_{2, t}\right] \mathrm{d} t, \\
\mathrm{~d} Y_{t} & =\left(r-\frac{1}{2} \operatorname{Tr}\left[\gamma_{1, t}+\gamma_{2, t}\right]\right) \mathrm{d} t+\operatorname{Tr}\left[\sqrt{\gamma_{1, t}} \mathrm{~d} Z_{t}^{\gamma_{1}}+\sqrt{\gamma_{2, t}} \mathrm{~d} Z_{t}^{\gamma_{2}}\right] .
\end{aligned}
$$

This can still be presented in the form as follows:

$$
\begin{aligned}
\mathrm{d} Y(t)= & \left(r-\frac{1}{2} \operatorname{Tr}\left[\gamma_{1, t}+\gamma_{2, t}\right]\right) \mathrm{d} t+\operatorname{Tr}\left[\sqrt{\gamma_{1, t}}\left(\mathrm{~d} W_{t}^{\gamma_{1}} R_{1}^{T}+\mathrm{d} B_{t}^{\gamma_{1}} \sqrt{\square-R_{1} R_{1}^{T}}\right)\right. \\
& \left.+\sqrt{\gamma_{2, t}}\left(\mathrm{~d} W_{t}^{\gamma_{2}} R_{2}^{T}+\mathrm{d} B_{t}^{\gamma_{2}} \sqrt{\square-R_{2} R_{2}^{T}}\right)\right], \quad Y_{0}=y .
\end{aligned}
$$


3.2.1. Infinitesimal Generator under Double Wishart Model. The log-price process and Wishart affine processes, combined with the corresponding pair of two correlated
Brownian motions, $Z_{s}^{\gamma_{1}, Y}, Z_{s}^{\gamma_{1}, \gamma_{1}}$ and $Z_{s}^{\gamma_{2}, Y}, Z_{s}^{\gamma_{2}, \gamma_{2}}$, can be presented in the form as in Benabid et al. [9] for easy handling of the dynamics.

$$
\begin{aligned}
\mathrm{d} Y_{t} & =\left(r-\frac{1}{2} \operatorname{Tr}\left[\gamma_{1, t}+\gamma_{2, t}\right]\right) \mathrm{d} t+\operatorname{Tr}\left[\sqrt{\gamma_{1, t}} \mathrm{~d} Z_{t}^{\gamma_{1}, Y}+\sqrt{\gamma_{2, t}} \mathrm{~d} Z_{t}^{\gamma_{2}, Y}\right] \\
\mathrm{d} \operatorname{Tr}\left(\gamma_{1, t}\right) & =\left(\left(\beta_{1} \operatorname{Tr}\left(Q_{1}^{T} Q_{1}\right)\right)+2 \operatorname{Tr}\left(M_{1} \gamma_{1, t}\right) \mathrm{d} t+2 \sqrt{\operatorname{Tr}\left(Q_{1}^{T} Q_{1} \gamma_{1, t}\right)}\left(\rho_{1, t} \mathrm{~d} Z_{t}^{\gamma_{1}, Y}+\sqrt{\square-\rho_{1, t}^{2}} \mathrm{~d} Z_{t}^{\gamma_{1}}\right)\right. \\
\mathrm{d} \operatorname{Tr}\left(\gamma_{2, t}\right) & =\left(\left(\beta_{2} \operatorname{Tr}\left(Q_{2}^{T} Q_{2}\right)\right)+2 \operatorname{Tr}\left(M_{2} \gamma_{2, t}\right)\right) \mathrm{d} t+2 \sqrt{\operatorname{Tr}\left(Q_{2}^{T} Q_{2} \gamma_{2, t}\right)}\left(\rho_{2, t} \mathrm{~d} Z_{t}^{\gamma_{2}, Y}+\sqrt{\square-\rho_{2, t}^{2}} \mathrm{~d} Z_{t}^{\gamma_{2}}\right),
\end{aligned}
$$

where

$$
\begin{aligned}
& d<Z^{\gamma_{1}, Y}, Z^{\gamma_{1}}>_{t}=\rho_{1, t}=\frac{\operatorname{Tr}\left(R_{1}^{T} Q_{1} \gamma_{1, t}\right)}{\sqrt{\operatorname{Tr}\left(\gamma_{1, t}\right)} \sqrt{\operatorname{Tr}\left(Q_{1}^{T} Q_{1} \gamma_{1, t}\right)}}, \\
& d<Z^{\gamma_{2}, Y}, Z^{\gamma_{2}}>_{t}=\rho_{2, t}=\frac{\operatorname{Tr}\left(R_{2}^{T} Q_{2} \gamma_{2, t}\right)}{\sqrt{\operatorname{Tr}\left(\gamma_{2, t}\right)} \sqrt{\operatorname{Tr}\left(Q_{2}^{T} Q_{2} \gamma_{2, t}\right)}} .
\end{aligned}
$$

Lemma 5. The infinitesimal generator under the double Wishart volatility model for $Y_{t}, \gamma_{1, t}$, and $\gamma_{2, t}$ is as follows:

$$
\begin{aligned}
\mathscr{L}_{Y, \gamma_{1}, \gamma_{2}}= & \left(r-\frac{\operatorname{Tr}\left[\gamma_{1}+\gamma_{2}\right]}{2}\right) \frac{\partial}{\partial y}+\frac{\operatorname{Tr}\left[\gamma_{1}+\gamma_{2}\right]}{2} \frac{\partial^{2}}{\partial y^{2}} \\
& +\left(\beta_{1} \operatorname{Tr}\left(Q_{1}^{T} Q_{1}\right)+2 \operatorname{Tr}\left(M_{1} \gamma_{1}\right)\right) \frac{\partial}{\partial \gamma_{1}}+2 \operatorname{Tr}\left(\gamma_{1} \frac{\partial}{\partial \gamma_{1}} Q_{1}^{T} Q_{1} \frac{\partial}{\partial \gamma_{1}}\right) \\
& +\left(\beta_{2} \operatorname{Tr}\left(Q_{2}^{T} Q_{2}\right)+2 \operatorname{Tr}\left(M_{2} \gamma_{2}\right)\right) \frac{\partial}{\partial \gamma_{2}}+2 \operatorname{Tr}\left(\gamma_{2} \frac{\partial}{\partial \gamma_{2}} Q_{2}^{T} Q_{2} \frac{\partial}{\partial \gamma_{2}}\right) \\
& +2 \operatorname{Tr}\left(\gamma_{1} R_{1} Q_{1} \frac{\partial}{\partial \gamma_{1}}\right) \frac{\partial}{\partial y}+2 \operatorname{Tr}\left(\gamma_{2} R_{2} Q_{2} \frac{\partial}{\partial \gamma_{2}}\right) \frac{\partial}{\partial y} .
\end{aligned}
$$

Proof. The infinitesimal generator has nontrivial term which arises from covariation $\left.d<\gamma_{\theta}^{i j}, Y\right\rangle$ corresponding to the coefficients of the term

$$
\frac{\partial^{2}}{\partial x_{\theta ; i j} \partial y}=\frac{\partial}{\partial x_{\theta ; i j}}\left(\frac{\partial}{\partial y}\right), \quad i, j=1, \ldots, n, \theta=1,2 .
$$

Let $C_{\theta: t}:=\sqrt{\gamma_{\theta: t}}$ be the square root matrix where

$$
\gamma_{\theta ; t}^{i j}=\sum_{t=1}^{n} C_{\theta ; t}^{i l} C_{\theta ; t}^{l j}=\sum_{t=1}^{n} C_{\theta ; t}^{i l} C_{\theta ; t}^{j l} .
$$

Since $C_{\theta: t}$ is symmetric, we determine the covariation terms corresponding to $\partial^{2} / \partial x_{\theta ; i j} \partial y$ coefficients as follows:

$$
\begin{aligned}
\left\langle\mathrm{d} \gamma_{\theta}^{i j}, Y\right\rangle & =\mathbb{E}_{t}\left[\left(\sum_{i, k=1}^{n} C_{\theta ; t}^{i l} d W_{l k}^{\theta} Q_{k j}^{\theta}+\sum_{l, k=1}^{n} C_{\theta ; t}^{j l} d W_{l k}^{\theta} Q_{k i}^{\theta}\right)\left(\sum_{l, k, h=1}^{n} C_{\theta ; t}^{l k} d W_{k h}^{\theta} R_{l h}^{\theta}\right)\right] \\
& =\sum_{l, k, h=1}^{n}\left(C_{\theta ; t}^{i l} Q_{k j}^{\theta}+C_{\theta ; t}^{j l} Q_{k i}^{\theta}\right) C_{\theta ; t}^{h l} R_{h k}^{\theta} \mathrm{d} t \\
& =\sum_{k, h=1}^{n}\left[\left(\sum_{l=1}^{n} C_{\theta ; t}^{i l} C_{\theta ; t}^{h l}\right) Q_{k j}^{\theta}+\left(\sum_{l=1}^{n} C_{\theta ; t}^{j l} C_{\theta ; t}^{h l}\right) Q_{k i}^{\theta}\right] R_{h k}^{\theta} \mathrm{d} t \\
& =\sum_{k, h=1}^{n}\left(\gamma_{\theta ; t}^{i h} Q_{k j}^{\theta}+\gamma_{\theta ; t}^{j h} Q_{k i}^{\theta}\right) R_{h k}^{\theta} \mathrm{d} t .
\end{aligned}
$$


This provides the corresponding coefficients of the term:

$$
2 \operatorname{Tr}\left(\gamma_{\theta} R^{\theta} Q^{\theta} D_{\theta}\right) \frac{\partial}{\partial y}=2 \sum_{i, j, k, h=1}^{n} D_{\theta}^{i j} \gamma_{\theta}^{j h} R_{h k}^{\theta} Q_{k i}^{\theta} \frac{\partial}{\partial y} .
$$

The notation when $\theta=1, \gamma_{1}=\gamma_{1}, R^{1}=R_{1}, Q^{1}=Q_{1}$, and $D_{1}=\partial / \partial x_{i j}^{\gamma_{1}}$ while for $\theta=2, \gamma_{2}=\gamma_{2}, R^{2}=R_{2}$, $Q^{2}=Q_{2}$ and $D_{2}=\partial / \partial x_{i j}^{\gamma_{2}}$.

3.3. The Euler-Maruyama Discretization Scheme. This section basically deals with the discretization scheme of the BiWishart volatility model using Euler-Maruyama discretization technique.

This is one of the best approximation methods to handle sophisticated stochastic differential equations as in the study by Ahdida and Alfonsi [6], Fadugba et al. [19], Dereich et al. [20], Berkaoui et al. [21], and Mao [22]. It is a time discrete approximation of an Ito process. Consider $S_{t}$ as an Ito process on $t \in\left[t_{0}, T\right]$ satisfying differential equation as follows:

$$
S_{t}=S_{0}+\int_{0}^{t} \mu(t, s) \mathrm{d} s+\int_{0}^{t} \gamma(t, s) \mathrm{d} W_{s}, \quad t \geq 0
$$

For discretization $t_{0}<t_{1}<t_{2} \cdots<t_{N}=T$ or on a regular grid $t_{i}=\mathrm{iT} / N$, it is given as

$$
\widehat{S}_{t_{i+1}}=\widehat{S}_{t_{i}}+\mu\left(\widehat{S}_{t_{i}}\right) \frac{T}{N}+\gamma\left(\widehat{S}_{t_{i}}\right)\left(W_{t_{i+1}}-W_{t_{i}}\right), \quad 1 \leq i \leq N-1,
$$

and also considering a continuous case, we have

$$
\widehat{S}_{t_{i+1}}=\widehat{S}_{t_{i}}+\mu\left(\widehat{S}_{t_{i}}\right)\left(t_{i+1}-t_{i}\right)+\gamma\left(\widehat{S}_{t_{i}}\right)\left(W_{t_{i+1}}-W_{t_{i}}\right), \quad t \in\left[t_{i}, t_{i+1}\right) .
$$

One can observe that $\widehat{S}$ the Euler scheme depends on $N$ throughout the discretization grid, and it is similarly better to denote it as $\widehat{S}^{N}$.

\subsection{The Corrected Euler-Maruyama Discretization Scheme for} Double Wishart Affine Processes. This section discusses the discretization techniques for the Bi-variate Wishart model. The log-price dynamic and Wishart processes are discretized using corrected Euler-Maruyama method. We notice that the Euler scheme is not well defined; in fact, the Gaussian increment may lead the scheme to negative values with some positive probability, and the square root is then no long defined. Therefore, this calls in the need to apply the corrected Euler-Maruyama scheme to avoid negative values (see Alfonsi [13] and Gauthier and Possamaï [23]).

The asset dynamic is as follows:

$$
\frac{\mathrm{d} S_{t}}{S_{t}}=\mu \mathrm{d} t+\operatorname{Tr}\left[\sqrt{\gamma}_{1, t} \mathrm{~d} Z_{t}^{\gamma_{1}}+\sqrt{\gamma}_{2, t} \mathrm{~d} Z_{t}^{\gamma_{2}}\right], \quad S_{0}=s,
$$

where the Wishart matrix processes are given as follows:

$$
\gamma_{j, t}=\gamma_{j, 0}+\int_{0}^{t}\left(\Omega_{j}+\eta_{j}(\gamma, s)\right) \mathrm{d} s+\int_{0}^{t} \sqrt{\gamma}_{j, s} \mathrm{~d} W_{s}^{\gamma_{j}} Q_{j}+\int_{0}^{t} Q_{j}^{T}\left(\mathrm{~d} W_{s}^{\gamma_{j}}\right)^{T} \sqrt{\gamma_{j, s}} \quad \text { for } j=1,2,
$$

where $\Omega_{j}=\beta_{j} Q_{j} Q_{j}^{T}$ such that $\gamma_{j, 0}, \Omega_{j} \in S_{n}^{+}(\mathbb{R})$ while $\eta_{j} \in \mathscr{L}\left(S_{n}^{+}(\mathbb{R})\right)$ and $M_{j} \in M_{n}(\mathbb{R})$.
Then, discretize equations (50) and (37), by considering a time horizon $T$ and regular time grid $t_{i}^{N}=i T / N$, for $i=0, \ldots, N$.

$$
\widehat{\gamma}_{j, t_{i+1}^{N}}=\widehat{\gamma}_{j, t_{i}^{N}}+\left(\Omega_{j}+\eta_{j}\left(\widehat{\gamma}_{j}, t_{i}^{N}\right)\right) \frac{T}{N}+\sqrt{\widehat{\gamma}_{j, t_{i}^{N}}^{+}}\left(W_{t_{i+1}^{N}}^{\gamma_{j}}-W_{t_{i}^{N}}^{\gamma_{j}}\right) Q_{j}+Q_{j}^{T}\left(W_{t_{i+1}^{N}}^{\gamma_{j}}-W_{t_{i}^{N}}^{\gamma_{j}}\right) \sqrt{\widehat{\gamma}_{j, t_{i}^{N}}^{+}} \text {, for } \quad j=1,2 \text {, }
$$

where $\widehat{\gamma}_{j, t_{0}^{N}}=\gamma_{j, 0}=\gamma_{j}$.

Now, we proceed to discretize the log-price dynamics as follows:

$$
\widehat{Y}_{t_{i+1}^{N}}^{y}=\widehat{Y}_{t_{i}^{N}}^{y}+\left(\mu-\frac{1}{2} \operatorname{Tr}\left[\widehat{\gamma}_{1, t_{i}^{N}}+\widehat{\gamma}_{2, t_{i}^{N}}\right]\right) \frac{T}{N}+\operatorname{Tr}\left[\sqrt{\widehat{\gamma}_{1, t_{i}^{N}}^{+}}\left(Z_{t_{i+1}^{N}}^{\gamma_{1}}-Z_{t_{i}^{N}}^{\gamma_{1}}\right)+\sqrt{\widehat{\gamma}_{2, t_{i}^{N}}^{+}}\left(Z_{t_{i+1}^{N}}^{\gamma_{2}}-Z_{t_{i}^{N}}^{\gamma_{2}}\right)\right] .
$$

We now discretize the Brownian motions in equation (52) by considering the correlation structures in (30), that is,

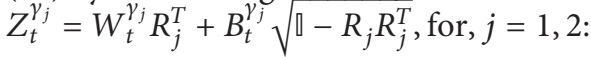




$$
\begin{aligned}
Z_{t_{i+1}^{N}}^{\gamma_{j}}-Z_{t_{i}^{N}}^{\gamma_{j}} & =W_{t_{i+1}^{N}}^{\gamma_{j}} R_{j}^{T}+B_{t_{i+1}^{N}}^{\gamma_{j}} \sqrt{\square-R_{j} R_{j}^{T}}-\left(W_{t_{i}^{N}}^{\gamma_{j}} R_{j}^{T}+B_{t_{i}^{N}}^{\gamma_{j}} \sqrt{\square-R_{j} R_{j}^{T}}\right) \\
& =\left(W_{t_{i+1}^{N}}^{\gamma_{j}}-W_{t_{i}^{N}}^{\gamma_{j}}\right) R_{j}^{T}+\left(B_{t_{i+1}^{N}}^{\gamma_{j}}-B_{t_{i}^{N}}^{\gamma_{j}}\right) \sqrt{\square-R_{j} R_{j}^{T}} .
\end{aligned}
$$

These Brownian matrices $Z^{\gamma_{j}}$ and $B^{\gamma_{j}}$ follow normal distribution, that is,

$$
\begin{aligned}
& W_{t_{i+1}^{N}}^{\gamma_{j}}-W_{t_{i}^{N}}^{\gamma_{j}} \sim \mathcal{N}\left(0,\left(t_{i+1}^{N}-t_{i}^{N}\right) \llbracket\right), \\
& W_{t_{i+1}^{N}}^{\gamma_{j}}-W_{t_{i}^{N}}^{\gamma_{j}} \sim \sqrt{t_{i+1}^{N}-t_{i}^{N}} \mathcal{N}(0, \llbracket),
\end{aligned}
$$

and

$$
B_{t_{i+1}^{N}}^{\gamma_{j}}-B_{t_{i}^{N}}^{\gamma_{j}} \sim \sqrt{t_{i+1}^{N}-t_{i}^{N}} \mathcal{N}(0, \llbracket)
$$

Hence, simplifying the computation, we substitute equations (54) and (55) into equation (54)

$$
Z_{t_{i+1}^{N}}^{\gamma_{j}}-Z_{t_{i}^{N}}^{\gamma_{j}} \sim\left(R_{j}^{T}+\sqrt{\square-R_{j} R_{j}^{T}}\right) \sqrt{t_{i+1}^{N}-t_{i}^{N}} \mathcal{N}(0, \mathbb{q}) .
$$

Equation (56) is reduced in the form as follows:

$$
Z_{t_{i+1}^{N}}^{\gamma_{j}}-Z_{t_{i}^{N}}^{\gamma_{j}} \sim\left(R_{j}^{T}+\sqrt{\square-R_{j} R_{j}^{T}}\right) \sqrt{\frac{T}{N}} \mathcal{N}(0, \llbracket) .
$$

Let the $\log$-price dynamics $Y_{t}=\log \left(S_{t}\right)$ in equation (52) be $\widehat{Y}_{t_{i+1}^{N}}=\log \left(\widehat{S}_{t_{i+1}^{N}}\right)$ and $\log \left(\widehat{S}_{t_{i}^{N}}\right)$ as follows:

$$
\log \left(\widehat{S}_{t_{i+1}^{N}}\right)=\log \left(\widehat{S}_{t_{i}^{N}}\right)+\left(\mu-\frac{1}{2} \operatorname{Tr}\left[\widehat{\gamma}_{1, t_{i}^{N}}+\widehat{\gamma}_{2, t_{i}^{N}}\right]\right) \frac{T}{N}+\operatorname{Tr}\left[\sqrt{\widehat{\gamma}_{1, t_{i}^{N}}^{+}}\left(Z_{t_{i+1}^{N}}^{\gamma_{1}}-Z_{t_{i}^{N}}^{\gamma_{1}}\right)+\sqrt{\widehat{\gamma}_{2, t_{i}^{N}}^{+}}\left(Z_{t_{i+1}^{N}}^{\gamma_{2}}-Z_{t_{i}^{N}}^{\gamma_{2}}\right)\right]
$$

This log-price expression can be represented in the form as follows:

$$
\begin{aligned}
\log \left(\widehat{S}_{t_{i+1}^{N}}\right)= & \log \left(\widehat{S}_{t_{i}^{N}}\right)+\mu \frac{T}{N}-\frac{1}{2} \int_{t_{i}^{N}}^{t_{i+1}^{N}} \operatorname{Tr}\left[\gamma_{1}(s)+\gamma_{2}(s)\right] \mathrm{d} s \\
& +\operatorname{Tr}\left[\int_{t_{i}^{N}}^{t_{i+1}^{N}} \sqrt{\gamma_{1}^{+}(s)} \mathrm{d} Z_{s}^{\gamma_{1}}+\int_{t_{i}^{N}}^{t_{i+1}^{N}} \sqrt{\gamma_{2}^{+}(s)} \mathrm{d} Z_{s}^{\gamma_{2}}\right] .
\end{aligned}
$$

The increment $\xi_{k, i 1 \leq k \leq \beta}$ allows to simulate $\operatorname{Tr}\left[\int_{t_{i}^{N}}^{t_{i+1}^{N}} \sqrt{\gamma_{1}(s)} \mathrm{d} W_{s}^{\gamma_{1}} R_{1}^{T}\right]$, and for other terms, the standard Euler scheme is selected, that is,

$$
\begin{aligned}
\int_{t_{i}^{N}}^{t_{i+1}^{N}} \sqrt{\gamma_{1}^{+}(s) \mathrm{d} W_{s}^{\gamma_{1}}} & =\sum_{k=1}^{\beta} \int_{t_{i}^{N}}^{t_{i+1}^{N}} X_{k, s} \mathrm{~d} W_{k, s}^{\gamma_{1}}=\sqrt{\Delta t} \sum_{k=1}^{\beta} X_{k, t} \xi_{k, i}^{T} \\
\operatorname{Tr}\left[\int_{t_{i}^{N}}^{t_{i+1}^{N}} \sqrt{\gamma_{1}^{+}(s)} \mathrm{d} B_{s}^{\gamma_{1}} \sqrt{\square-R_{1} R_{1}^{T}}\right] & =\sqrt{\Delta t} \sqrt{\operatorname{Tr}\left(\widehat{\gamma}_{1, t_{i}^{N}}^{+}\left(\square-R_{1} R_{1}^{T}\right)\right)} \zeta_{1, i} .
\end{aligned}
$$

We observe that the integral term in equation (59) needs to be simplified as follows:

$$
\begin{aligned}
\int_{t_{i}^{N}}^{t_{i+1}^{N}} \sqrt{\gamma_{1}^{+}(s) \mathrm{d} Z_{s}^{\gamma_{1}}} & =\int_{t_{i}^{N}}^{t_{i+1}^{N}} \sqrt{\gamma_{1}^{+}(s) \mathrm{d} W_{s}^{\gamma_{1}} R_{1}^{T}}+\int_{t_{i}^{N}}^{t_{i+1}^{N}} \sqrt{\gamma_{1}^{+}(s) \mathrm{d} B_{s}^{\gamma_{1}}} \sqrt{\square-R_{1} R_{1}^{T}} \\
& =\sqrt{t_{i+1}^{N}-t_{i}^{N}} \sum_{k=1}^{l} \widehat{X}_{1}\left(k, t_{i}^{N}\right) \xi_{k, i}^{T} R_{1}^{T}+\sqrt{t_{i+1}^{N}-t_{i}^{N}} \sqrt{\widehat{\gamma}_{1, t_{i}^{N}}^{+}\left(\square-R_{1} R_{1}^{T}\right)} \zeta_{1, i} \\
& =\sqrt{\frac{T}{N}} \sum_{k=1}^{l} \hat{X}_{1}\left(k, t_{i}^{N}\right) \xi_{k, i}^{T} R_{1}^{T}+\sqrt{\frac{T}{N}} \sqrt{\widehat{\gamma}_{1, t_{i}^{N}}^{+}\left(\square-R_{1} R_{1}^{T}\right)} \zeta_{1, i},
\end{aligned}
$$


where the Gaussian vector $\zeta_{1, i} \sim \mathcal{N}(0, \llbracket)$ and the same for the second term are as follows:

$$
\int_{t_{i}^{N}}^{t_{i+1}^{N}} \sqrt{\gamma_{2}^{+}(s)} \mathrm{d} Z_{s}^{\gamma_{2}}=\sqrt{\frac{T}{N}} \sum_{k=1}^{l} \widehat{X}_{2}\left(k, t_{i}^{N}\right) \xi_{k, i}^{T} R_{2}^{T}+\sqrt{\frac{T}{N}} \sqrt{\widehat{\gamma}_{2, t_{i}^{N}}^{+}\left(\square-R_{2} R_{2}^{T}\right)} \zeta_{2, i},
$$

where $\zeta_{2, i} \sim \mathcal{N}(0, \square)$, and then we can substitute equations

(61) and (62) in equation (59) as follows:

$$
\begin{aligned}
\log \left(\frac{\widehat{S}_{t_{i+1}^{N}}}{\widehat{S}_{t_{i}^{N}}}\right)= & \mu \frac{T}{N}-\frac{1}{2} \int_{t_{i}^{N}}^{t_{i+1}^{N}} \operatorname{Tr}\left[\gamma_{1}(s)+\gamma_{2}(s)\right] \mathrm{d} s \\
& +\sqrt{\frac{T}{N}} \operatorname{Tr}\left[\sum_{k=1}^{l} \hat{X}_{1}\left(k, t_{i}^{N}\right) \xi_{k, i}^{T} R_{1}^{T}+\sum_{k=1}^{l} \hat{X}_{2}\left(k, t_{i}^{N}\right) \xi_{k, i}^{T} R_{2}^{T}\right] \\
& +\sqrt{\frac{T}{N}}\left[\sqrt{\operatorname{Tr}\left(\hat{\gamma}_{1, t_{i}^{N}}^{+}\left(\square-R_{1} R_{1}^{T}\right)\right)} \zeta_{1, i}+\sqrt{\operatorname{Tr}\left(\hat{\gamma}_{2, t_{i}^{N}}^{+}\left(\square-R_{2} R_{2}^{T}\right)\right)} \zeta_{2, i}\right] .
\end{aligned}
$$

Finally, we obtain the Euler-Maruyama discretization scheme for the double Wishart diffusion processes as follows:

$$
\begin{aligned}
& \log \left(\frac{\widehat{S}_{t_{i+1}^{N}}}{\widehat{S}_{t_{i}^{N}}}\right)=\left(\mu-\frac{1}{2} \operatorname{Tr}\left[\widehat{\gamma}_{1, t_{i}^{N}}+\widehat{\gamma}_{2, t_{i}^{N}}\right]\right) \frac{T}{N}+\sqrt{\frac{T}{N}} \operatorname{Tr}\left[\sum_{k=1}^{l} \widehat{X}_{1}\left(k, t_{i}^{N}\right) \xi_{k, i}^{T} R_{1}^{T}+\sum_{k=1}^{l} \widehat{X}_{2}\left(k, t_{i}^{N}\right) \xi_{k, i}^{T} R_{2}^{T}\right] \\
& +\sqrt{\frac{T}{N}}\left[\sqrt{\operatorname{Tr}\left(\hat{\gamma}_{1, t_{i}^{N}}^{+}\left(\square-R_{1} R_{1}^{T}\right)\right)} \zeta_{1, i}+\sqrt{\operatorname{Tr}\left(\hat{\gamma}_{2, t_{i}^{N}}^{+}\left(\square-R_{2} R_{2}^{T}\right)\right)} \zeta_{2, i}\right] . \\
& \gamma_{1,0}=\left(\begin{array}{cc}
0.00008 & 0 \\
0 & 0.000095
\end{array}\right) \text {, } \\
& \gamma_{2,0}=\left(\begin{array}{cc}
0.00012 & 0 \\
0 & 0.0001
\end{array}\right) \text {, } \\
& Q_{1}=\left(\begin{array}{cc}
0.10 & 0 \\
0 & 0.23
\end{array}\right) \text {, } \\
& Q_{2}=\left(\begin{array}{cc}
0.20 & 0 \\
0 & 0.25
\end{array}\right) \text {, }
\end{aligned}
$$

\section{Numerical Illustrations}

This section deals with numerical examples to examine the effects of volatility and other model parameters on the log-asset returns under the double Wishart volatility model.

4.1. The Log-Asset Returns under Double Wishart Volatility Model. The volatility specification under the double Wishart volatility model makes it mathematically flexible, to influence the asset return behavior. We quote market data drawn from QQQ (a fund by Invesco that tracks the performance of the stocks listed under the NASDAQ Index) options, April 2020, which has been used in Naryongo et al. [17]. We take the estimated parameter values from the market data, the variance matrices $\gamma_{1}$ and $\gamma_{2}$, with volatility matrices $Q_{1}$ and $Q_{2}$ are considered since they are very necessary parameters in a stochastic volatility model. with strike price $K=189$ and interest rate $r=0.05$.

Take the correlation values of

$$
\begin{aligned}
& R_{1}=\left(\begin{array}{ll}
-0.25 & 0.1 \\
-0.35 & 0.3
\end{array}\right), \\
& R_{2}=\left(\begin{array}{ll}
-0.5 & -0.60 \\
0.18 & -0.45
\end{array}\right) .
\end{aligned}
$$




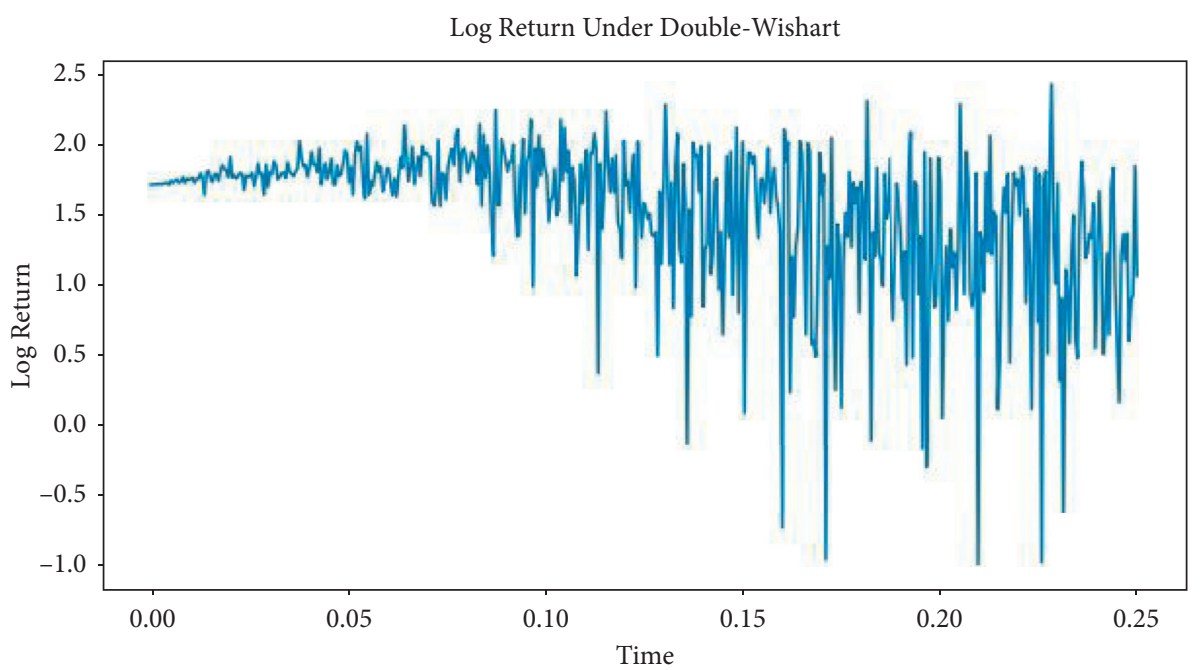

FIgURE 1: The log-asset return behaviors under the double Wishart volatility model at 3 months.

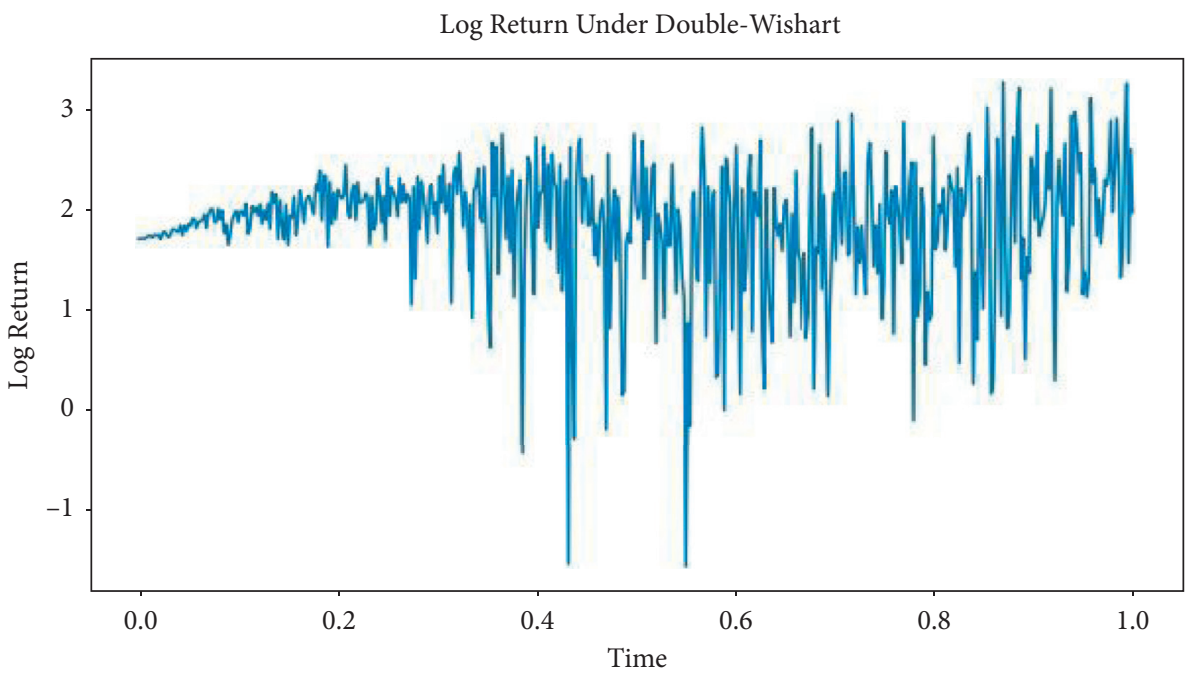

FIgURE 2: The log-asset returns trend under the double Wishart model when $N=500$ at one year.

Figure 1 demonstrates the effect of implied volatilities and correlation matrices on the log-asset returns under the double Wishart model. Given the parameter values, we observe that the log-asset returns under the double Wishart model exhibit interesting behaviors. This depends also on the choice of the model parameters, such as $\beta_{1}=4, \beta_{2}=3$, and the number of paths $N=500$. This example proves that the double Wishart volatility model has greater flexibility. The log-returns under the double Wishart model are more volatile towards the end of the trading period. The asset prices increase from the higher deviations with the number of paths under trading from the values of volatility matrices. It leads to higher returns in 3-month asset trading period under the double Wishart process.
Figure 2 shows that a change in correlation matrices affects the behavior of the asset returns, that is, when

$$
\begin{aligned}
& R_{1}=\left(\begin{array}{cc}
0.0 & 0.85 \\
-0.05 & 0.75
\end{array}\right), \\
& R_{2}=\left(\begin{array}{cc}
0.20 & 0.0 \\
0.01 & -0.20
\end{array}\right),
\end{aligned}
$$

this provides different stock price behavior patterns due to flexibility in the model parameters. This can help investors to study and predict the behavior of stock price over time. The parameters $\beta_{1}=4$ and $\beta_{2}=3$ are maintained. The stock price trend for one year is under double Wishart model, when $N=500$; the asset price increases with time from the 


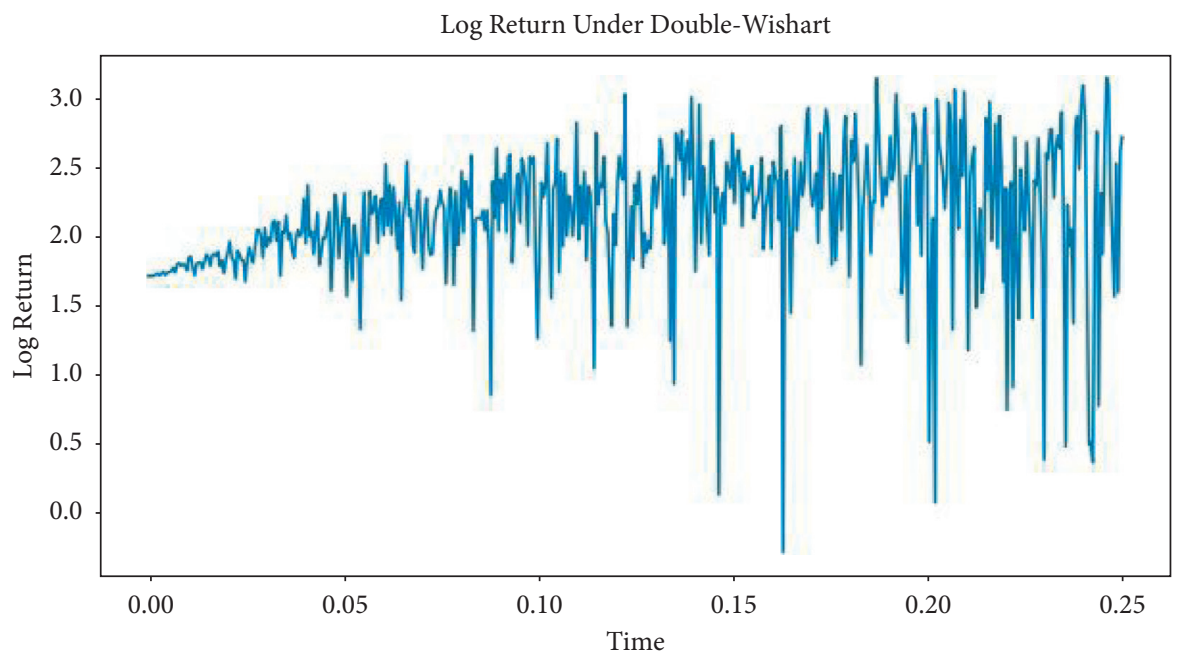

FIgURE 3: The log-returns behavior under the double Wishart model at 3 months with different correlation matrices.

implied volatility as it approaches annual trading period from the volatility matrices.

Figure 3 illustrates the effect of correlation matrices and $N=500$ on the log-asset returns trend under the double Wishart model while considering parameters $\beta_{1}=4$ and $\beta_{2}=3$, while taking the values of

$$
\begin{aligned}
& R_{1}=\left(\begin{array}{cc}
0.0 & 0.05 \\
0.05 & 0.6
\end{array}\right), \\
& R_{2}=\left(\begin{array}{cc}
0.8 & 0.5 \\
0.45 & 0.65
\end{array}\right) .
\end{aligned}
$$

The model shows interesting stock price trend predictions. We note that the model parameters influence the stock prices or log-returns greatly due to mathematical flexibility of the model. We can observe that there is higher asset returns at the end of the trading period.

\section{Conclusion}

The multifactor Heston model with two dependence matrices, that is, the double Wishart stochastic volatility model is flexible enough to describe the market prices. This solves the problem of pricing financial derivatives or assets in short or long maturities. The effect of the model parameter specification influences the behavior of the asset returns over time. This provides a theoretical framework for investors to study the market behavior over time. The numerical illustrations show the effect of model parameters on the asset return behavior under double Wishart model. We recommend future work on the exact simulation method and higher-order discretization techniques for the double Wishart volatility model.

\section{Appendix}

\section{Verification of Brownian Motions in Lemma 3}

The following processes $X_{t}^{\gamma_{1}}, X_{t}^{\gamma_{2}}, \xi_{t}$, and $\eta_{t}$ are Brownian motions.

Proof.

$$
\begin{aligned}
\operatorname{Cov}_{t}\left(\mathrm{~d} X_{t}^{\gamma_{1}}, \mathrm{~d} X_{t}^{\gamma_{1}}\right) & =\mathbb{E}_{t}\left[\left(\mathrm{~d} X_{t}^{\gamma_{1}}\right)\left(\mathrm{d} X_{t}^{\gamma_{1}}\right)^{T}\right] \\
& =\mathbb{E}_{t}\left[\frac{\operatorname{Tr}\left(\sqrt{\gamma_{1, t}} \mathrm{~d} Z_{t}^{\gamma_{1}}\right)}{\sqrt{\operatorname{Tr}\left(\gamma_{1, t}\right)}} \frac{\operatorname{Tr}\left(\sqrt{\gamma_{1, t}} \mathrm{~d} Z_{t}^{\gamma_{1}}\right)}{\sqrt{\operatorname{Tr}\left(\gamma_{1, t}\right)}}\right] \\
& =\frac{\sum_{i, j} \operatorname{Cov}_{t}\left(e_{i}^{T} \sqrt{\gamma_{1, t}} \mathrm{~d} Z_{t}^{\gamma_{1}} e_{i}, e_{j}^{T} \sqrt{\gamma_{1, t}} \mathrm{~d} Z_{t}^{\gamma_{1}} e_{j}\right)}{\sqrt{\operatorname{Tr}\left(\gamma_{1, t}\right)} \sqrt{\operatorname{Tr}\left(\gamma_{1, t}\right)}} \\
& =\frac{\sum_{i, j} e_{i}^{T} \sqrt{\gamma_{1, t}} \operatorname{Tr}\left(e_{i} e_{j}^{T}\right) \sqrt{\gamma_{1, t}} e_{j} \mathrm{~d} t}{\operatorname{Tr}\left(\gamma_{1, t}\right)} \\
& =\frac{\sum_{i, j} \operatorname{Tr}\left(e_{i} e_{j}^{T}\right) e_{i}^{T} \gamma_{1, t} e_{j} \mathrm{~d} t}{\operatorname{Tr}\left(\gamma_{1, t}\right)}=\frac{\sum_{i, j} e_{j}^{T} e_{i} e_{i}^{T} \gamma_{1, t} e_{j} \mathrm{~d} t}{\operatorname{Tr}\left(\gamma_{1, t}\right)} \\
& =\frac{\sum_{j} e_{j}^{T} \gamma_{1, t} e_{j} \mathrm{~d} t}{\operatorname{Tr}\left(\gamma_{1, t}\right)}=\frac{\operatorname{Tr}\left(\gamma_{1, t}\right) \mathrm{d} t}{\operatorname{Tr}\left(\gamma_{1, t}\right)} \\
& =\mathrm{d} t .
\end{aligned}
$$

Similarly, for Brownian motion $X_{t}^{\gamma_{2}}$, its proof can be obtained through the same procedures as above. 

follows.

Then, the authors show that $\xi_{t}$ is a Brownian motion as Proof.

$$
\begin{aligned}
\operatorname{Cov}_{t}\left(\mathrm{~d} \xi_{t}, \mathrm{~d} \xi_{t}\right) & =\mathbb{E}_{t}\left[\frac{\operatorname{Tr}\left(Q \mathrm{~d} W_{t}^{\gamma_{1}} \sqrt{\gamma_{1, t}}\right)}{\sqrt{\operatorname{Tr}\left(Q^{T} Q \gamma_{1, t}\right)}} \frac{\operatorname{Tr}\left(Q \mathrm{~d} W_{t} \sqrt{\gamma_{1, t}}\right)}{\sqrt{\operatorname{Tr}\left(Q^{T} Q \gamma_{1, t}\right)}}\right] \\
& =\frac{\sum_{i, j} \operatorname{Cov}_{t}\left(e_{i}^{T} Q \mathrm{~d} W_{t}^{\gamma_{1}} \sqrt{\gamma_{1, t}} e_{i}, e_{j}^{T} Q \mathrm{~d} W_{t}^{\gamma_{1}} \sqrt{\gamma_{1, t}} e_{j}\right)}{\sqrt{\operatorname{Tr}\left(Q^{T} Q \gamma_{1, t}\right)} \sqrt{\operatorname{Tr}\left(Q^{T} Q \gamma_{1, t}\right)}} \\
& =\frac{\sum_{i, j} e_{i}^{T} \sqrt{\gamma_{1, t}} \operatorname{Tr}\left(Q^{T} e_{i}, e_{j}^{T} Q\right) \sqrt{\gamma_{1, t}} e_{j} \mathrm{~d} t}{\sqrt{\operatorname{Tr}\left(Q^{T} Q \gamma_{1, t}\right)} \sqrt{\operatorname{Tr}\left(Q^{T} Q \gamma_{1, t}\right)}} \\
& =\frac{\sum_{i, j} \operatorname{Tr}\left(Q^{T} Q e_{i} e_{j}^{T}\right) e_{i}^{T} \gamma_{1, t} e_{j} \mathrm{~d} t}{\operatorname{Tr}\left(Q^{T} Q \gamma_{1, t}\right)} \frac{\sum_{i, j} e_{j}^{T} Q^{T} Q e_{i} e_{i}^{T} \gamma_{1, t} e_{j} \mathrm{~d} t}{\operatorname{Tr}\left(Q^{T} Q \gamma_{1, t}\right)} \\
& =\frac{\sum_{i, j} e_{j}^{T} Q^{T} Q \gamma_{1, t} e_{j} \mathrm{~d} t}{\operatorname{Tr}\left(Q^{T} Q \gamma_{1, t}\right)}=\frac{\operatorname{Tr}\left(Q^{T} Q \gamma_{1, t}\right) \mathrm{d} t}{\operatorname{Tr}\left(Q^{T} Q \gamma_{1, t}\right)} \\
& =\mathrm{d} t .
\end{aligned}
$$

Similarly, the same procedures can be followed to show that $\eta_{t}$ is a Brownian motion.

\section{Data Availability}

The data used to support the findings of this study are available from the corresponding author upon request.

\section{Conflicts of Interest}

The authors in this paper declare that they have no conflicts of interest with regard to the publication of this paper.

\section{Acknowledgments}

The authors extend their gratitude to the Pan African University Institute for Basic sciences, Technology and Innovation (Pausti) at Jomo Kenyatta University (JKUAT).

\section{References}

[1] F. Black and M. Scholes, "The pricing of options and corporate liabilities," Journal of Political Economy, vol. 81, no. 3, pp. 637-654, 1973.

[2] S. L. Heston, "A closed-form solution for options with stochastic volatility with applications to bond and currency options," Review of Financial Studies, vol. 6, no. 2, pp. 327-343, 1993.

[3] D. Filipovic and E. Mayerhofer, "Affine diffusion processes: theory and applications," Advanced Financial Modelling, vol. 8, pp. 1-40, 2009.

[4] J. Da Fonseca, M. Grasselli, and C. Tebaldi, "A multifactor volatility heston model," Quantitative Finance, vol. 8, no. 6, pp. 591-604, 2008.
[5] P. Christoffersen, S. Heston, and K. Jacobs, "The shape and term structure of the index option smirk: why multifactor stochastic volatility models work so well," Management Science, vol. 55, no. 12, pp. 1914-1932, 2009.

[6] A. Ahdida and A. Alfonsi, "Exact and high-order discretization schemes for wishart processes and their affine extensions," The Annals of Applied Probability, vol. 23, no. 3, pp. 1025-1073, 2013.

[7] C. Kang, W. Kang, and J. M. Lee, "Exact simulation of the wishart multidimensional stochastic volatility model," $O p$ erations Research, vol. 65, no. 5, pp. 1190-1206, 2017.

[8] C. Gouriéroux, "Continuous time wishart process for stochastic risk," Econometric Reviews, vol. 25, no. 2-3, pp. 177-217, 2006.

[9] A. Benabid, H. Bensusan, and N. El Karoui, Wishart Stochastic Volatility: Asymptotic Smile and Numerical Framework, 2008.

[10] C. Kang and W. Kang, Exact Simulation of Wishart Multidimensional Stochastic Volatility Model, https://arxiv.org/abs/ 1309.0557, 2013.

[11] M.-F. Bru, "Wishart processes," Journal of Theoretical Probability, vol. 4, no. 4, pp. 725-751, 1991.

[12] J. Da Fonseca, M. Grasselli, and C. Tebaldi, "Wishart multidimensional stochastic volatility, to appear as: a multifactor volatility heston model' in quantitative finance," Mimeo Ecole Supérieure d' Ingénieurs Léonard de Vinci, vol. 8, 2005.

[13] A. Alfonsi, Affine Diffusions and Related Processes: Simulation, Springer, Berlin, Germany, 2015.

[14] C. Gourieroux and R. Sufana, "Derivative pricing with wishart multivariate stochastic volatility: application to credit risk," SSRN E-Library, vol. 28, pp. 1-44, 2004.

[15] G. La Bua and D. Marazzina, "Calibration and advanced simulation schemes for the wishart stochastic volatility model," Quantitative Finance, vol. 19, no. 6, pp. 997-1016, 2019. 
[16] T. Björk, Arbitrage Theory in Continuous Time, Oxford university Press, Oxford, UK, 2009.

[17] R. Naryongo, P. Ngare, and A. Waititu, "European option pricing under wishart processes," Journal of Mathematics, vol. 2021, Article ID 7411885, 24 pages, 2021.

[18] S. E. Shreve, Stochastic Calculus for Finance II: ContinuousTime Models, Springer Science \& Business Media, Berlin, Germany, 2004.

[19] S. Fadugba, B. Adegboyegun, and O. Ogunbiyi, "On the convergence of euler maruyama method and milstein scheme for the solution of stochastic differential equations," International Journal of Applied Mathematics and Modeling, vol. 1, 2013.

[20] S. Dereich, A. Neuenkirch, and L. Szpruch, "An Euler-type method for the strong approximation of the Cox-IngersollRoss process," Proceedings of the Royal Society A: Mathematical, Physical and Engineering Sciences, vol. 468, no. 2140, pp. 1105-1115, 2012.

[21] A. Berkaoui, M. Bossy, and A. Diop, "Euler scheme for sdes with non-lipschitz diffusion coefficient: strong convergence," ESAIM: Probability and Statistics, vol. 12, pp. 1-11, 2008.

[22] X. Mao, "The truncated Euler-Maruyama method for stochastic differential equations," Journal of Computational and Applied Mathematics, vol. 290, pp. 370-384, 2015.

[23] P. Gauthier and D. Possamaï, Efficient Simulation of the Wishart Model, 2009. 